\title{
Operating Room Leadership and Management
}

\author{
Alan D. Kaye, Charles J. Fox III, Richard D. Urman. Cambridge University Press, 2012. \\ ISBN 978-1-107-01753-5
}

Jennifer Szerb, MD

Received: 25 March 2013/Accepted: 3 April 2013/Published online: 16 April 2013

(C) Canadian Anesthesiologists' Society 2013

As a self-employed Canadian anesthesiologist who admits to having an "us versus them" view of health administrators, reading Operating Room Leadership and Management has been an educational experience. This text is an excellent and thought-provoking reference for physicians considering leadership roles in the hospital or physicians frustrated with operating room (OR) inefficiencies.

Both the editors and contributors of this textbook practice in the United States; hence, chapters on billing or operating an ambulatory surgery centre as a successful business would not apply to the Canadian reader. Nevertheless, Section 1 of the book is universally germane, with Appendix A, "Characteristics of Leadership", being particularly useful. As a witness to bullying and other inappropriate behaviour in the OR, I particularly enjoyed the analysis of behaviours according to the concept of "game theory".

The strategic planning process is discussed in chapter 3 , and I disagree with the authors' opinion that the development of vision and mission statements should be a topdown process imposed by senior leaders. In my view, organizations are strengthened by using a grassroots process, which furthers cooperation towards a common goal.

"Must-read" sections in this textbook include chapters 5 and 6. Coming from a hospital where the budgetary deficit was dealt with by selective OR closures, I learned in "Health care and economic realities" that surgical patients in the United States generate the majority of revenue for most hospitals. This reality highlights the need for major changes in how remuneration for surgical procedures is generated in Canadian hospitals, while accounting for the

J. Szerb, MD ( $\square)$

Dalhousie University, Halifax, NS, Canada

e-mail: Jennifer.Szerb@cdha.nshealth.ca challenges presented by an academic health sciences centre with more complex patients, longer operative times, and additional learners.

As Canadian anesthesiologists, we are under increasing pressure to reduce induction and emergence times. At the same time, we must focus on the prevention of medical errors and provide quality care. Chapter 6 eloquently sets out the economics of operating room efficiency, particularly the impact of overruns in operating room time on labour costs. The author of this chapter points out that a three to nine-minute reduction in the average turnover time results in only a $0.8-1.8 \%$ reduction in labour cost. There is a paramount need to look at surgeon-specific operating times based on accurate OR information systems and to schedule these cases appropriately into the allotted time available. This task has not yet been implemented in my institution despite a significant expenditure on external efficiency consultants.

In chapter 7, there is an excellent discussion of process capacity and the occurrence of bottlenecks. When planning for the appropriate number of staff, consideration must be given to the movement of patients through all stages, i.e., from registration through to the postanesthesia care unit (PACU). The author shows how a simple calculation of capacity can predict where bottlenecks will occur. In Table 7.2, the bottleneck arises in the PACU, simply due to the duration of each patient's stay. This example validates my own attempts to reduce PACU stays in order to lessen the delays that unfortunately remain a significant problem in my institution.

Anesthesiologists are frequently called upon to provide input into future OR planning and renovation. Chapters 8 and 9 are particularly helpful; I was struck by the innovative concept of an instrument preparation room attached to the OR where personnel can set up the equipment as the 
patient is emerging. Key to this concept is ensuring that the ventilation is in plenum relative to the OR, which means there is no atmospheric contamination from the OR. This would also circumvent the need for an induction room, so there would be no need to move the patient during a period of potential instability.

The chapters on preoperative evaluation and postanesthesia care were somewhat basic; however, this textbook is also written for non-anesthesiologists. The concept of Web-based preoperative assessment tools was most interesting, as it may facilitate advanced triaging of patients and a more accurate assessment of American Society of Anesthesiologists' classification than the use of standardized forms administered over the phone.

Reading a book, like going on vacation, can make a person more appreciative of home. Chapter 14 describes the current anesthesia environment in the United States where 16 states have opted out of a federal requirement for physician supervision of anesthetists. The remaining states have a varying mix of certified registered nurse anesthetist/ anesthesia assistant/physician, and as a result, one anesthesiologist may be called upon to supervise five or more concurrent anesthesia procedures. Decisions on the mix are driven not only by clinical needs but also by the economics of the hospital.

In summary, this innovative book encompasses all aspects of OR management. For Canadian anesthesiologists, the chapters I have highlighted warrant reading in their entirety. The profession will be inspired to make new and creative changes to improve OR efficiency and anesthesia practice and will be thankful they practice medicine in Canada.

Competing interests None declared. 